\title{
MINIMAL DEPENDENT SETS
}

\author{
D. S. ASCHE \\ (Received 10 June 1965)
}

\section{Introduction}

The subject matter of this note is the notion of a dependence structure on an abstract set. There are a number of different approaches to this topic and it is known that many of these lead to precisely the same structure ${ }^{1}$. Axioms are given here to specify the minimal dependent sets for such a structure. They are closely related to conditions introduced by Hassler Whitney in [1] and to a certain "elimination axiom" given by A. P. Robertson and J. D. Weston in [2]. Theorem 1 shows that a dependence structure may equally well be defined by means of axioms for the independent sets. Axiom (I) is adapted from a condition due to R. Rado [3]. Theorem 2 links our minimal dependent sets with Whitney's "circuits". Theorem $\mathbf{3}$ is an "elimination" theorem which generalizes the statement of our axiom $\left(\mathrm{C}_{2}\right)$. Theorem 4 is due to Rado ([3], Theorem 3, p. 307), and A. W. Ingleton [4]. It is shown here to follow from Theorem 3.

\section{Axioms and theorems}

Let $X$ be a set. Let $\mathscr{C}$ be a set of non-empty finite subsets of $X$. Furthermore, let $\mathscr{C}$ satisfy the following two conditions.

$\left(\mathrm{C}_{1}\right)$ No proper subset of a member of $\mathscr{C}$ is a member of $\mathscr{C}$.

$\left(C_{2}\right)$ If $E$ and $F$ are distinct members of $\mathscr{C}$ and $x \in E \cap F$, then $E \cup F$ has a subset belonging to $\mathscr{C}$ but not containing $x$.

Axiom $\left(\mathrm{C}_{2}\right)$ is the "elimination axiom" of Robertson and Weston [2] who apply it to a set $\mathscr{R}$ of finite subsets of a set $X$ and use no other condition. For the case where the empty set is not a member of $\mathscr{R}$, it can be seen that the members of $\mathscr{C}$ are precisely the minimal members of $\mathscr{R}$, for it is clear that the "elimination axiom" must hold with $\mathscr{R}$ replaced by the set of its minimal members. In [2] the authors define "pure sets" as those non-empty subsets of $X$ which fail to contain members of $\mathscr{R}$. Here we define independent sets to be those subsets of $X$ which fail to contain

1 A study of the various axioms for a dependence structure formed the topic of a M. Sc. thesis by the author at Monash University. 
members of $\mathscr{C}$. The "pure sets" are then precisely the non-empty independent sets. The following theorem characterizes these independent sets.

THEOREM 1. A set $\mathscr{U}$ of subsets of a set $X$ is the set of independent sets defined by a set $\mathscr{C}$ of non-empty finite subsets of $X$ satisfying $\left(\mathrm{C}_{1}\right)$ and $\left(\mathrm{C}_{2}\right)$ if, and only if $\mathscr{U}$ is non-empty, $\mathscr{U}$ has the inductive property and $\mathscr{U}$ satisfies the following condition.

(I) If $A$ and $B$ are subsets of $X$ such that $A \notin \mathscr{U}, B \notin \mathscr{U}$, and $A \cap B \in \mathscr{U}$, then for all elements $x \in A \cup B$ it follows that $(A \cup B) \backslash\{x\} \notin \mathscr{U}$.

Proof. Firstly, let $\mathscr{U}$ be the set of independent sets. Then $\mathscr{U}$ is nonempty since $\square \in \mathscr{U}$, where $\square$ is the empty set. Now if $A \in \mathscr{U}$, then clearly every subset of $A$ belongs to $\mathscr{U}$. If $A \notin \mathscr{U}$, then a member of $\mathscr{C}$ contained in $A$ is a finite subset of $A$ which fails to belong to $\mathscr{U}$. Thus $\mathscr{U}$ has the inductive property. To verify condition (I) let $A \notin \mathscr{U}, B \notin \mathscr{U}, A \cap B \in \mathscr{U}$, $x \in A \cup B$. Then there exist sets $C \in \mathscr{C}, D \in \mathscr{C}$ such that $C \leqq A, D \subseteq B$. Also $C \neq D$, since $A \cap B \in \mathscr{U}$. If $x \notin C \cap D$, then either $C \subseteq(A \cup B) \mid\{x\}$ or $D \subseteq(A \cup B) \backslash\{x\}$ and so $(A \cup B) \backslash\{x\} \notin \mathscr{U}$. If $x \in C \cap D$, then from $\left(\mathrm{C}_{2}\right)$ there exists $E \in \mathscr{C}$ with $E \subseteq(C \cup D) \backslash\{x\} \subseteq(A \cup B) \backslash\{x\}$ so again $(A \cup B) \backslash\{x\} \notin \mathscr{U}$. Thus condition (I) holds. One may observe that the set $\mathscr{C}$ is precisely the set of subsets of $X$ minimal with respect to not belonging to $\mathscr{U}$.

Secondly, let $\mathscr{U}$ be a set of subsets of $X$ having the properties stated in the theorem, and let $\mathscr{C}$ be the set of subsets of $X$ minimal with respect to not belonging to $\mathscr{U}$. Since $\mathscr{U}$ is non-empty and possesses the inductive property, it follows that $\square \in \mathscr{U}$. Hence $\square \notin \mathscr{C}$. Also from the inductive property, any subset not in $\mathscr{U}$ contains a finite subset not in $\mathscr{U}$. Hence $\mathscr{C}$ consists of non-empty finite sets. That $\left(C_{1}\right)$ holds is clear from the definition of $\mathscr{C}$. In order to verify $\left(C_{2}\right)$, let $E \in \mathscr{C}, F \in \mathscr{C}, E \neq F$, and $x \in E \cap F$. Then $E \notin \mathscr{U}, F \notin \mathscr{U}$ and also $E \cap F \in \mathscr{U}$ because $E \cap F$ is a proper subset of $E$. It follows from condition $(\mathrm{I})$ that $(E \cup F) \mid\{x\} \notin \mathscr{U}$. Then since it is a finite set, we have that $(E \cup F) \backslash\{x\}$ must contain a member of $\mathscr{C}$. To complete the proof of the theorem one obsesves that the set $\mathscr{U}$ consists precisely of those subsets of $X$ which fail to contain members of $\mathscr{C}$.

In the paper $[1]$, Whitney uses the following axiom $\left(\mathrm{C}_{2}^{\prime}\right)$ together with $\left(\mathrm{C}_{1}\right)$ and refers to the members of $\mathscr{C}$ by the name "circuits". Also he restricts attention to the case where the set $X$ is finite.

$\left(\mathrm{C}_{2}^{\prime}\right)$ If $E$ and $F$ are distinct members of $\mathscr{C}$, if $x \in E \cap F$ and if $y \in E \backslash F$, then $E \cup F$ has a subset belonging to $\mathscr{C}$ which contains $y$ but fails to contain $x$.

Since $\left(\mathrm{C}_{2}^{\prime}\right)$ seems to impose a stronger condition on the set $\mathscr{C}$ than $\left(\mathrm{C}_{2}\right)$, the following theorem may be of some interest. In any case it provides the link between the two systems. 
THEOREM 2. If $\mathscr{C}$ is a set of non-empty finite subsets of a set $X$, then conditions $\left(\mathrm{C}_{1}\right)$ and $\left(\mathrm{C}_{2}\right)$ together are equivalent to conditions $\left(\mathrm{C}_{1}\right)$ and $\left(\mathrm{C}_{2}^{\prime}\right)$ together.

Proof. It is clear that $\left(C_{2}\right)$ follows from $\left(C_{2}^{\prime}\right)$. Suppose now that $\left(C_{1}\right)$ and $\left(\mathrm{C}_{2}\right)$ hold but that $\left(\mathrm{C}_{2}^{\prime}\right)$ fails to hold and let $m$ be the least integer such that for some pair of sets $E$ and $F$ belonging to $\mathscr{C}$ and satisfying $|E \cup F|=m$ there exist elements $x \in E \cap F$ and $y \in E \backslash F$ such that $E \cup F$ contains no member $G$ of $\mathscr{C}$ satisfying $y \in G$ and $x \notin G$. By supposition such an integer exists, and we may assume that $E, F, x$ and $y$ have the stated properties. Then by $\left(\mathrm{C}_{2}\right)$ there exists a subset $G$ of $E \cup F$ belonging to $\mathscr{C}$ and failing to contain $x$. But then $y \notin G$. From $\left(C_{1}\right)$ we may choose $z \in G \mid E$, and then using the fact that $|G \cup F|<m$ and the minimality of $m$ we may apply $\left(\mathrm{C}_{2}^{\prime}\right)$ to the sets $G$ and $F$ and the elements $z \in G \cap F$ and $x \in F \mid G$. Thus there exists a subset $H$ of $G \cup F$ belonging to $\mathscr{C}$ and containing $x$ but not containing $z$. But then $|E \cup H|<m$ since $z \notin E \cup H$ and we may apply $\left(\mathrm{C}_{2}^{\prime}\right)$ to the sets $E$ and $H$ and the elements $x \in E \cap H$ and $y \in E \backslash H$ to show the existence of a subset $J$ of $E \cup H$ belonging to $\mathscr{C}$, containing $y$ and failing to contain $x$. This, however, is a contradiction.

Theorem 3. If $A_{1}, A_{2}, \cdots, A_{n}$ are members of $\mathscr{C}$, if $n \geqq 2$ and if

$$
A_{i} \ddagger \bigcup\left\{A_{j}: j<i\right\}, \quad i=2, \cdots, n
$$

holds, then for each subset $B$ of $X$ with $|B|=r<n$, there exist members $C_{1}, C_{2}, \cdots, C_{n-r}$ of $\mathscr{C}$ such that

$$
\begin{aligned}
& C_{i} \subseteq \bigcup\left\{A_{k}: k=1, \cdots, n\right\} \mid B \text { and } \\
& C_{i} \nsubseteq \bigcup\left\{C_{j}: j \neq i\right\} \text { hold for } i=1,2, \cdots, n-r .
\end{aligned}
$$

Proof. Let $(n, r)$ denote the case of the theorem for which $n$ members of the set $\mathscr{C}$ are considered and the set $B$ consists of $r$ elements.

Case $(n, 0)$. By the hypothesis and since $\square \notin \mathscr{C}$ we may choose $x_{1} \in A_{1}$ and elements $x_{i} \in A_{i} \backslash \bigcup\left\{A_{j}: j<i\right\}$ for $i=2, \cdots, n$. The following sets $B_{i, j}$ are defined for the indices $j=i+1, \cdots, n$ and $i=1,2, \cdots, n$. For $j=2, \cdots, n$, if $x_{1} \notin A_{j}$ put $B_{1, j}=A_{j}$ and if $x_{1} \in A_{j}$ using $\left(\mathrm{C}_{2}^{\prime}\right)$ choose $B_{1, j} \in \mathscr{C}$ such that $B_{1, j} \subseteq A_{1} \cup A_{j}, x_{1} \notin B_{1, j}$, and $x_{j} \in B_{1, j}$. Then by induction, if $x_{i} \notin B_{i-1, j}$ define $B_{i, j}=B_{i-1, j}$ and if $x_{i} \in B_{i-1, j}$ choose $B_{i, j} \in \mathscr{C}$ such that $B_{i, j} \subseteq B_{i-1, i} \cup B_{i-1, j}, x_{i} \notin B_{i, j}$ and $x_{j} \in B_{i, j}$ where the choice is made possible by $\left(\mathrm{C}_{2}^{\prime}\right)$ and by the fact that $x_{i} \in B_{i-1, j} \cap B_{i-1, i}$ and $x_{j} \in B_{i-1, j} \mid B_{i-1, i}$. Then let $C_{1}=A_{1}, C_{2}=B_{1,2}, \cdots, C_{n}=B_{n-1, n}$. Then for $i=1, \ldots, n$ we have $x_{i} \in C_{i}$ and $x_{i} \notin \bigcup\left\{C_{j}: j=i+1, \cdots, n\right\}$. Also one observes that for $i=2, \cdots, n$ we have $x_{i} \notin \bigcup\left\{C_{j}: j<i\right\}$. Thus for $i=1, \cdots, n$ the relation $C_{i} \notin \bigcup\left\{C_{j}: j \neq i\right\}$ holds. Finally it is clear that 
$C_{i} \subseteq \bigcup\left\{A_{k}: k=1, \cdots, n\right\}$ must also hold and hence the sets $C_{1}, \cdots, C_{n}$ satisfy the requirements of the theorem in this case.

Case $(n, r)$. This case of the theorem is shown to depend on the case $(n-1, r-1)$ and so ultimately on the case $(n-r, 0)$ which is a case already proved. Let $B \subseteq X$ and $|B|=r$. By the case $(n, 0)$ it may be assumed that the sets $A_{1}, \cdots, A_{n}$ already satisfy the relation $A_{i} \neq \bigcup\left\{A_{j}: j \neq i\right\}$. Let $x \in B$. If $x \notin \bigcup\left\{A_{i}: i=1, \cdots, n\right\}$, the case reduces immediately to the case $(n-1, r-1)$. Otherwise, by the symmetry it may be assumed that $x \in A_{n}$. Now choose elements $y_{i} \in A_{i} \backslash \bigcup\left\{A_{j}: j \neq i\right\}$ for $i=1,2, \cdots, n-1$. Using $\left(\mathrm{C}_{2}^{\prime}\right)$ where necessary, there exist members $B_{1}, B_{2}, \cdots, B_{n-1}$ of $\mathscr{C}$ such that for $j=1,2, \cdots, n-1$ we have $B_{j} \subseteq A_{n} \cup A_{j}, x \notin B_{j}$ and $y_{j} \in B_{j}$. One then notices that $y_{j} \notin \bigcup\left\{B_{i}: i \neq j\right\}$ and so $B_{j} \notin \bigcup\left\{B_{i}: i \neq j\right\}$ for $j=1, \cdots, n-1$. Applying the theorem for the case $(n-1, r-1)$ to these members $B_{1}, \cdots, B_{n-1}$ of $\mathscr{C}$ and to the set $B \backslash\{x\}$ we obtain members $C_{1}, C_{2}, \cdots, C_{n-r}$ of $\mathscr{C}$ having the required properties.

Theorem 4. If $A_{1}, A_{2}, \cdots, A_{n}$ are subsets of $X$ which do not belong to the set $\mathscr{U}$ of independent sets, and if for $i=2,3, \cdots, n$ the sets $A_{i} \cap \bigcup\left\{A_{j}: j<i\right\}$ belong to $\mathscr{U}$, then for any subset $B$ of $X$ with $|B|<n$ it follows that $\bigcup\left\{A_{i}: i=1, \cdots, n\right\} \mid B$ is not a member of $\mathscr{U}$.

Proof. Let $B_{1}, B_{2}, \cdots, B_{n}$ be minimal dependent sets (i.e. members of $\mathscr{C}$ ) contained in $A_{1}, A_{2}, \cdots, A_{n}$ respectively. Then for $i=1,2, \cdots, n$ we have

$$
B_{i} \cap \bigcup\left\{B_{j}: j<i\right\} \subseteq A_{i} \cap \bigcup\left\{A_{j}: j<i\right\} .
$$

Then since $B_{i} \notin \mathscr{U}$ it follows that $B_{i} \notin \bigcup\left\{B_{j}: j<i\right\}$ for $i=2,3, \cdots, n$. By theorem 3, if $|B|=r$, there are $(n-r)>0$ minimal dependent sets contained in $\bigcup\left\{A_{i}: i=1,2, \cdots, n\right\} \mid B$ and hence this latter set is not a member of $\mathscr{U}$.

\section{References}

[1] Hassler Whitney, 'On the abstract properties of linear dependence', Amer. J. Math. 57 (1935), $509-533$.

[2] A. P. Robertson and J. D. Weston, 'A general basis theorem', Proc. Edinburgh Math. Soc. (2) 11 (1958-1959), 139-141.

[3] R. Rado, 'Note on independence functions', Proc. London Math. Soc. 7 (1957), 300-320.

[4] A. W. Ingleton, 'A note on independence functions and rank', J. London Math. Soc. 34 (1959), 49-56.

Department of Mathematics

Monash University

Clayton, Victoria 